International Journal of Maternal and Child Health and AIDS (2020), Volume 9, Issue I, I4-2 I

\begin{tabular}{ll}
\hline & INTERNATIONAL JOURNAL of \\
& MATERNAL and CHILD HEALTH and AIDS \\
& ISSN 216I-864X (Online) \\
& ISSN 216I-8674 (Print) \\
IJMA Available online at www.mchandaids.org & DOI: 10.21 I 06/ijma.290 \\
\hline
\end{tabular}

ORIGINALARTICLE

\title{
Maternal Caffeine Consumption and Racial Disparities in Fetal Telomere Length
}

\author{
Isabel Griffin, MPH;' Boubakari Ibrahimou, PhD;' Natasha Navejar, BS; ${ }^{2}$ Anjali Aggarwal, MD; ${ }^{3}$ \\ Kristopher Myers, MPH;' Daniel Mauck, MPH;' Korede K.Yusuf, MBBS, PhD; ${ }^{4}$ Usman J.Wudil, MD, MPH; 5 \\ Muktar H.Aliyu, MD, DrPH; ${ }^{5}$ Hamisu M. Salihu, MD, PhD ${ }^{2 \bowtie}$ \\ 'Florida International University, Robert Stempel College of Public Health and Social Work, Department of Epidemiology, I I 200 SW 8 ${ }^{\text {th }}$ Street \#500, \\ Miami, Florida 33174, USA; ${ }^{2}$ Center of Excellence in Health Equity, Training and Research, Baylor College of Medicine, One Baylor Plaza, MS:4II Houston, \\ TX 77030, USA; ${ }^{3}$ Department of Family and Community Medicine, Baylor College of Medicine, 370I Kirby Drive, Houston, Texas, 77098, USA; ${ }^{4}$ Adelphi \\ University, College of Nursing and Public Health, One South Avenue, Garden City, NY I I530, USA; ${ }^{5}$ Vanderbilt University Medical Center, Vanderbilt Insti- \\ tute for Global Health, 2525 West End Ave, Suite 750, Nashville, TN 37203, USA \\ Corresponding author email: hamisu.salihu@bcm.edu
}

\section{ABSTRACT}

Background and Objectives: The identification of risk factors for shorter telomere length, especially during fetal development, would be important towards caffeine consumption recommendations for pregnant women on a global scale. The purpose of this study was to evaluate the association between caffeine intake and fetal telomere length as well as racial/ethnic differences in telomere length regardless of maternal caffeine consumption status.

Methods: Caffeine intake was measured using a food frequency questionnaire (FFQ). Three generalized linear models (GLM) were compared based on binary categorical variables of caffeine levels using data mean value of II7.3 mg as cut-off; the World Health Organization (WHO) recommendations of $300 \mathrm{mg}$; and the American College of Obstetricians and Gynecologists (ACOG) recommendations of $200 \mathrm{mg}$. The association between caffeine consumption and telomere length (telomere to single-copy [T/S] ratio) was then assessed.

Results: Among 57 maternal-fetal dyads, 77.2\% reported less than $200 \mathrm{mg}$ of caffeine (ACOG) and 89.5\% less than $300 \mathrm{mg}$ (WHO). Both WHO and ACOG models found that caffeine intake was significantly and positively associated with longer telomere length $(p<0.05)$; and sodium $(p<0.05)$. Other" race $(p<0.00 \mathrm{I})$ and "white" race $(p<0.00 \mathrm{I})$ were also significantly and positively associated with longer telomere length in the same models. Increasing maternal age shortened telomere length significantly in all models $(p<0.00 \mathrm{I})$.

Conclusion and Global Health implications: Caffeine intake, maternal age, and race may be associated with alterations in fetal telomere length. This indicates that caffeine consumption during pregnancy may have long-term implications for fetal development. The racial/ethnic differences in telomere length found in this study warrant larger studies to further confirm these associations.

Key words: • Telomere • Fetal telomere length • Caffeine • Pregnancy • Maternal-Fetal medicine - Racial/ethnic differences

Copyright (C) 2020 Griffin et al. Published by Global Health and Education Projects, Inc. This is an open-access article distributed under the terms of the Creative Commons Attribution License CC BY 4.0. 


\section{Introduction}

Telomeres are DNA-based regions that cap the end of chromosomes to prevent degradation of essential genes involved in cell replication. Telomeres progressively shorten, and cells become susceptible to degradation as the shortening occurs, which is the result of low telomerase levels (an enzyme that elongates chromosomes by adding TTAGGG sequences).' As telomere length can strongly predict chromosomal aging and long-term health outcomes, researchers have aimed to explore the association between dietary factors and telomere length. Caffeine, a stimulant found in foods (tea, coffee, soft drinks, chocolate, and over-the-counter medications), is believed to influence telomere length at a cellular level. Evident in laboratory studies conducted with yeast, caffeine appears to act through the phosphoinositide 3-kinase-related protein kinases Tell and MEcl, which are known regulators of telomere length. ${ }^{2}$ Several studies provide contradictory findings regarding the influence of caffeine on telomere length. In a study by Liu et al. among 4,780 female nurses who reported coffee consumption, the investigators found that telomere length increased with elevated levels of caffeine consumption. ${ }^{3}$ However, in a separate study by Tucker et al., researchers found that for each $100 \mathrm{mg}$ of caffeine consumed, telomeres were 35.4 base pairs shorter. ${ }^{4}$ This same study found that coffee consumption within this population was positively associated with longer telomere length. ${ }^{4}$ These inconsistent findings indicate the need to continue examining the association while taking into account additional confounding factors as well as population subgroups, such as pregnant women, where caffeine consumption may be detrimental to the development of a healthy fetus. ${ }^{5}$

A major gap in knowledge is the impact of caffeine consumption within a pregnant population. During pregnancy, caffeine clearance from the mother's blood slows down significantly. A study by Wierzejeska et al. found that caffeine consumed by a pregnant woman passes through the placenta to the fetus freely. ${ }^{6}$ In another study by Torstein et al., researchers found that high caffeine intake in the third trimester may be a risk factor for fetal growth restriction, in particular, if the fetus is a boy. Current recommendations from the World Health Organization (WHO) advise pregnant women to limit total caffeine consumption to less than $300 \mathrm{mg}$ per day. ${ }^{8}$ The American Congress of Obstetricians and Gynecologists (ACOG) reported that moderate caffeine consumption of less than $200 \mathrm{mg}$ per day during pregnancy does not appear to be a risk factor for miscarriage or preterm births. ${ }^{9}$ Caffeine restrictions during pregnancy are based on several studies that have shown poor fetal outcomes, such as low birth weight babies and spontaneous abortions resulting from high caffeine intake prenatally. ${ }^{8-11}$ The identification of risk factors for shorter telomere length, especially during fetal development, would provide important information essential for making caffeine consumption recommendations on a global scale. In addition to the unknown influence of caffeine consumption on fetal telomere length, several studies have found that certain factors are associated with shorter telomere lengths. Previous research found that maternal-perceived stress during pregnancy was associated with shorter telomere length.' However, there is paucity of data on the differential effects of caffeine consumption by race/ethnicity.

\section{I. Aims and Hypothesis}

The purposes of this study were to: (I) examine the association between caffeine consumption during the third trimester of pregnancy and fetal telomere while taking into account potential confounders including maternal depression, maternal age, and sodium consumption; (2) examine this association with respect to the $\mathrm{WHO}$ and the ACOG recommendations regarding caffeine consumption during pregnancy; and (3) assess racial/ethnic differences in telomere length regardless of maternal caffeine consumption status.

\section{Materials and Methods}

A prospective cohort study was conducted by the University of South Florida (USF) Morsani College of Medicine from July 20II to September 2012 The study enrolled pregnant women in labor at Tampa General Hospital (TGH) in Tampa, Florida, USA. TGH admits patients for delivery who are socioeconomically disadvantaged, racially/ethnically 
diverse, and primarily uninsured or insured with Medicaid. Inclusion criteria for study participants required being over the age of 18 , with a primary language of English or Spanish, and delivering full-term single live birth with no congenital malformations. A food history questionnaire (FHQ) was provided to the study participants, which was completed before hospital discharge. Informed consent was obtained, and the study was approved by the institutional review boards at USF and TGH.'

\section{I. Cord Blood Telomere Ratio}

Telomere length and DNA extraction were performed by the USF Global Health Infectious Disease Research Laboratory. Laboratory personnel were blinded to all sociodemographic, health status, and birth outcome data. At birth, umbilical cord blood was collected from full-term infants. Because of the scarcity of the amount of genomic DNA obtained and the sample size, each individual sample was run in triplicate to determine the relative telomere to single-copy gene (T/S) ratio. Genomic DNA was isolated from a $200-\mu \mathrm{L}$ buffy-coat layer using the Qiagen DNeasy Blood and Tissue Kit. Isolated DNA was quantified with Nanodrop 2000c (Thermo Scientific, Waltham, MA, USA) and stored at $4^{\circ} \mathrm{C}$. The relative telomere lengths were then quantified by a comparison of the cycle threshold obtained by quantitative polymerase chain reaction (qPCR) cycle threshold $\left(\Delta \mathrm{C}_{\mathrm{t}}\right)$ values for the tell gene and reference single-copy gene, 36B4. Each cord blood sample was performed in triplicate. The relative telomere lengths in the DNA samples (telomere to single-copy number [T/S] ratio) were determined from cord blood by dividing the $C_{t}$ values of the absolute copy of the single gene 36B4.' Participants with T/S ratios greater than three and negative values were excluded as these values were considered non-informative outliers.

\subsection{Variables of Interest}

Sociodemographic data and newborn measurements were obtained from electronic medical records. Newborn characteristics included mean gestational age (weeks) and mean head circumference $(\mathrm{cm})$. Variables of interest used in this study included maternal age in years $(20-24,25-29,30-35,36$
- 39, 40 - 44), race/ethnicity (White, Black, Hispanic, Asian, Other), insurance status type (Medicaid/No Insurance, Private), marital status (Single, Married), number of previous pregnancies $(<2$ vs. $\geq 2$ ), and body mass index (<18.5 [Underweight], $\geq 18.5-$ $\leq 25$ [Normal], $>25-<30$ [Overweight], vs. $\geq 30$ [Obese]). Telomere length was categorized based on the mean T/S ratio $(\leq 0.86 \mathrm{I}$ vs. $>0.86 \mathrm{I})$. We defined dietary caffeine level $(\mathrm{mg})$ using the mean level of the study sample $(\leq \mid 27.2$ vs. $>\mid 27.2)$, the WHO caffeine recommendation $(\leq 300$ vs. $>300)$, and ACOG caffeine recommendation ( $\leq 200$ vs. $>200)$. Caffeine intake was measured using the Dietary History Questionnaire-I (DHQ), a food frequency questionnaire (FFQ) developed by the Risk Factor Monitoring and Methods Branch (RFMMB) of the National Cancer Institute $(\mathrm{NCl}) .^{12}$ The DHQ consisting of 124 food items, which include portion size and dietary supplement questions, is a validated tool that provides reasonable nutrient estimates. $^{12-14}$ The questionnaire addressed caffeine consumption by asking about coffee, iced tea, and hot tea consumption behaviors, and choosing between decaffeinated and caffeinated versions of these beverages. These responses were used to calculate the levels of caffeine consumption. It takes about an hour to complete the questionnaire, and analysis of the data was performed with the National Cancer Institute's Diet*Calc software developed based on the DHQ nutrient database. ${ }^{15-17}$ The nutrient and food group database was framed on the national dietary intake data from the 1994-96 U.S. Department of Agriculture's (USDA) Continuing Survey of Food Intake by Individuals (CSFII). ${ }^{17}$

\subsection{Data Analysis}

Descriptive statistics were used to describe study variables. A generalized linear model (GLM) was utilized to examine the relationship between caffeine level and telomere length. Caffeine consumption was evaluated within the model as a dichotomous variable. Three GLMs were compared based on the categorical variable of serum caffeine levels using means from the data as cut-off; WHO recommendation, and ACOG recommendation. The final GLMs consisted of mean T/S ratio ( $\geq 0.899$ vs. $<0.899$ ), included serum caffeine levels in $\mathrm{mg}$ (mean 
II7.29 or WHO recommendation 300 or ACOG recommendation 200), sodium (mean 4I27.8 mg), maternal age (mean 25.45), depression diagnosis (yes or no), and race/ethnicity (White, Hispanic, Asian, Black, and Other). Descriptive statistics were calculated in SAS 9.4 (Cary, NC: SAS Institute Inc; 20I4.), while the GLM analysis was conducted in $R$ version 3.3.I (Vienna,Austria; R Core Team; 20I7).

\section{Results}

Fifty-seven (59.4\%) of 96 maternal-fetal dyads with their corresponding fetal T/S ratios were included in this analysis, and pairs with T/S ratios greater than three or negative were excluded. The mean maternal age was 25.5 years $(S D=5.19$, range 18 - 39). Among the mothers, $35.7 \%$ were White; $30.4 \%$ were Black; $25.0 \%$ were Hispanic, and $8.9 \%$ were Other. About $\mathbf{8 0 . 7 \%}$ of the study population was covered by private health insurance, and $19.3 \%$ were on Medicaid or uninsured. Regarding marital status, $71.9 \%$ of the women were single, and $28.1 \%$ were married.About $71.9 \%$ of the mothers reported two or fewer previous pregnancies. The mean blood caffeine level (mg) was I I 7.3 (range 0 -940.9).Among the mothers, $77.2 \%$ had a caffeine level of less than $200 \mathrm{mg}$, consistent with ACOG recommendations, and $89.5 \%$ had a blood caffeine level of less than $300 \mathrm{mg}$, consistent with WHO recommendations. The mean gestational age of the newborns at delivery was 39.5 weeks (standard deviation $=1.3 \mathrm{I}$, range 37 - 42). All newborns were delivered during the third trimester. The mean newborn birthweight was 338I.4 grams (range $2 \mathrm{IOI}$ - 45I0) (Table I).

Three GLMs were run to fit the data in $R$ using three different cut-off points for caffeine (sample mean, WHO recommendation, and ACOG recommendation) and T/S ratios. The first GLM using mean caffeine consumption (Figure I) did not find a statistically significant association between the mean $\mathrm{T} / \mathrm{S}$ ratio and mean maternal caffeine level. However, both GLM models that included ACOG and WHO recommendations cut-off points for caffeine found that caffeine was statistically associated with longer telomere lengths $(p<0.05)$ (Figure 2). Older maternal age $(p<0.001)$ was significantly associated with shorter telomere length, whereas sodium intake $(p<0.05)$ "white" race $(p<0.00 \mathrm{I})$ and "other" race $(p<0.00 \mathrm{I})$ were associated with longer telomere length (Figure 2).

\section{Discussion}

This study suggests that high maternal caffeine intake and other factors including race, may be associated with increased fetal telomere length, whereas advanced maternal age may be associated with shortened fetal telomere lengths. The finding that caffeine food history questionnaire dietary levels were positively associated with telomere length suggests that caffeine consumption during pregnancies may have long term implications from a chromosomal perspective. It is unclear why certain races/ethnicities were associated with longer telomere length. However, there may be certain health disparities within certain racial populations that may predispose infants to premature chromosomal aging. Drury et al. found significant differences in telomere length between African American infants who weighed significantly less than white infants at birth, yet had significantly longer telomere length than White infants $(p=0.0 \mathrm{I})$, with the strongest association observed in Black female infants. ${ }^{18}$ In addition, research has also found that high caffeine intake during the third trimester of pregnancy may be a risk for fetal growth retardation, in particular, if the fetus is male. ${ }^{7}$ However, the gender of the fetus was not provided in our study, and as such we were unable to examine the role of fetal gender on telomere length.

We found that maternal age may be associated with shorter telomeres. This is consistent with the literature, which has reported that maternal age is weakly associated with telomere terminal restriction fragment (TRF) length in white blood cells in newborns. ${ }^{19}$ In addition, Prescott et al. found that paternal age, which was not available for this study, may be associated with fetal telomere length. ${ }^{20}$ However, both paternal and maternal ages may be confounded by one another as a mother may be more likely to choose a partner close to her age.

Based on the results of this study, if the sample population were to be increased to include babies with congenital defects and those who were excluded 
Table I: Demographic and developmental characteristics of mother and newborn at time of delivery ( $n=57$ maternal-newborn pairs)

\begin{tabular}{|c|c|}
\hline \multirow[t]{2}{*}{ Maternal Characteristics } & Study Population $(n=57)$ \\
\hline & Mean, standard deviation (range) $n(\%)$ \\
\hline Maternal Age at delivery (years) & $25.5,5.2(18-39)$ \\
\hline \multicolumn{2}{|l|}{ Race/Ethnicity } \\
\hline White & $20(35.7 \%)$ \\
\hline Black & $17(30.4 \%)$ \\
\hline Hispanic & $14(25.0 \%)$ \\
\hline Other & $5(8.9 \%)$ \\
\hline \multicolumn{2}{|l|}{ Health Insurance Status } \\
\hline Private & $46(80.7 \%)$ \\
\hline Medicaid /No Insurance & II (19.3\%) \\
\hline \multicolumn{2}{|l|}{ Marital Status } \\
\hline Married & $16(28.07 \%)$ \\
\hline Single & $4 \mathrm{I}(7 \mathrm{l} .9 \%)$ \\
\hline Gravidity & $2.19,1.65(1-10)$ \\
\hline$\leq 2$ & $4 \mid(71.9 \%)$ \\
\hline$>2$ & $16(28.1 \%)$ \\
\hline Maternal Weight & $91.5,25.6(54.4-189.6)$ \\
\hline Body Mass Index $\left(\mathrm{kg} / \mathrm{m}^{2}\right)$ & $34.7(48.9-67.5)$ \\
\hline Normal/Underweight & $14(24.8 \%)$ \\
\hline Overweight & $10(17.5 \%)$ \\
\hline Obese & $33(57.9 \%)$ \\
\hline \multicolumn{2}{|l|}{ Depression Status } \\
\hline Yes & $48(92.3 \%)$ \\
\hline No & $4(7.7 \%)$ \\
\hline Blood Caffeine Levels (mg) & I I7.3, $192.5(0-940.9)$ \\
\hline \multicolumn{2}{|l|}{ ACOG Recommendations } \\
\hline$>200 \mathrm{mg}$ & $13(22.8 \%)$ \\
\hline$\leq 200 \mathrm{mg}$ & $44(77.2 \%)$ \\
\hline \multicolumn{2}{|l|}{ WHO Recommendations } \\
\hline$>300 \mathrm{mg}$ & $6(10.5 \%)$ \\
\hline$\leq 300 \mathrm{mg}$ & $51(89.5 \%)$ \\
\hline Sodium (mg) & $4 \mid 27.8(43|.8-| 7263)$ \\
\hline \multicolumn{2}{|l|}{ Newborn Characteristics } \\
\hline Gestational Age (weeks) & $39.5,1.3(37-42)$ \\
\hline Birth Weight (grams) & $338 \mathrm{I} 4,509.1(2101-45 \mathrm{I} 0)$ \\
\hline T/S Ratio & $0.9,0.2(0.4-1.8)$ \\
\hline
\end{tabular}

*One individual was missing race; Five individuals were missing depression status

for complications, we might have detected a stronger association between caffeine and telomere length. The association between sodium and telomere length, in addition to caffeine, suggests the need for future studies to examine the role of diet during the third trimester of pregnancy on fetal telomere length. 


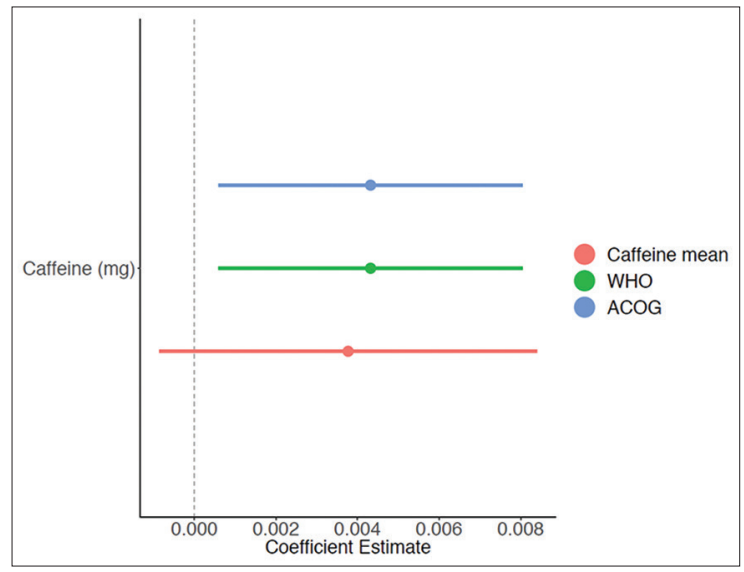

Figure I: Regression Coefficients of Caffeine Effect on Telomere Length Stratified by Cutoff Point for Caffeine (Sample Mean, WHO Recommendation, and ACOG Recommendation)

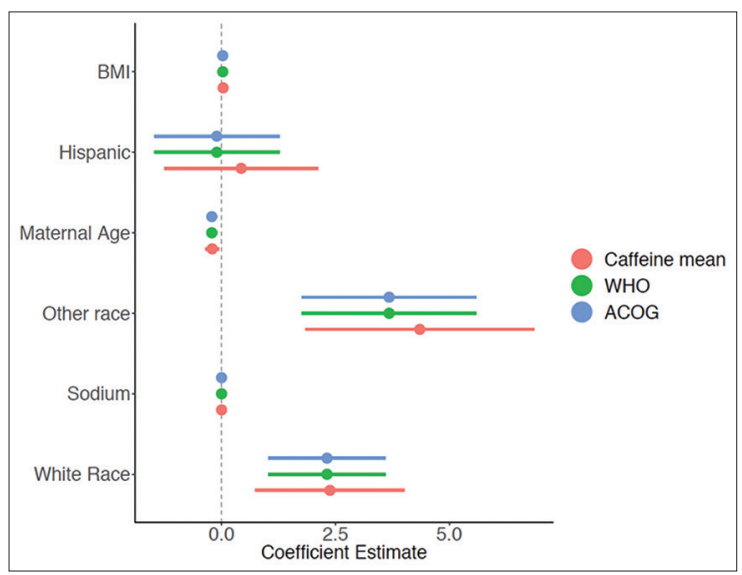

Figure 2: Regression Coefficients of Other Variable Effects on Telomere Length Stratified by Cutoff Point for Caffeine (Sample Mean,WHO Recommendation, and ACOG Recommendation)

\section{I. Limitations}

A limitation of this study is the possible selection bias in how study participants were recruited and included in the study. At-risk births (e.g., those with congenital malformations) were excluded from this study, which could have resulted in bias in the findings of this report as those births might have been more likely to have shorter telomere length (the outcome of interest) which would have probably impacted our current findings. Furthermore, given that only mothers with single births were included in this study, as such it is unknown whether multiple births may affect the findings. The participants of the study were also recruited from a low-income area of Tampa, Florida, as Tampa General Hospital often admits patients for delivery who are socioeconomically disadvantaged and primarily uninsured or insured with Medicaid; however, the women who met the inclusion criteria reported private insurance. Women who did not have access to prenatal care might not have received current recommendations regarding caffeine consumption during pregnancy, which may affect their caffeine consumption behaviors. Limited education levels among participants might also have played a role in understanding the different sources of caffeine in beverages, foods, and medications.

We were also unable to examine caffeine levels within fetal umbilical cord blood to strengthen the association that women with high levels of caffeine in their blood passed it to the fetus via the placenta, which could be a more direct measure of association between fetal exposure to caffeine and telomere length. However, in the future, statistical methods could be used to derive actual maternal bioavailability, which could minimize this limitation. In addition, data were only provided on caffeine exposures during the third trimester of pregnancy, although a better capture of caffeine exposure would have covered the first and second trimester as well. Nonetheless, this study bears several areas of strength including the elucidation of the association of race/ethnicity with telomere length over and beyond the caffeinetelomere length relationship.

\section{Conclusion and Global Health Implications}

This study identifies the need for further research to examine the association between fetal telomere length and caffeine consumption during pregnancy (i.e., fetal and maternal caffeine serum levels). Higher caffeine intake and certain racial categories may have a protective effect on fetal telomere length, suggesting that caffeine consumption during pregnancy may have long-term implications for fetal development. This study reinforced the literature in that maternal age was shown to have a significant association with shortened fetal telomere length. 
The racial/ethnic differences in telomere length found in this study warrant the conduct of larger studies to further delineate these associations.

The relationship between caffeine consumption and telomere length promises to be an avenue for exploring the impact of coffee consumption on pregnancy outcomes. Apart from water and tea, coffee is the third most consumed drink in the world. ${ }^{21}$ The findings in this study are, therefore, of global importance because it provides additional information that could inform future recommendations of prenatal caffeine consumption at the global level.

\section{Compliance with Ethical Considerations}

Conflicts of Interest: The authors have no conflicts of interest to report. Financial Disclosure: The authors have no financial disclosures to declare. Funding/Support: This work was supported by the James and EstherKing Biomedical Research Program, Florida Department of Health (grant number 4KB03 \& IKGI4-33987), and the University of South Florida Neuroscience Collaborative - Seed Grant Program and the University of South Florida Internal Awards Program Proposal Enhancement Grant.The funding agencies did not have any role in the conduct of the study, and the authors have no financial conflicts of interest. Ethical Approval: This study received IRB approval from the University of South Florida,Tampa.

\section{Key Messages}

- High maternal caffeine intake and other factors, including race, may be associated with longer fetal telomere length, whereas older maternal age may be associated with shorter fetal telomere length.

- The significant association between dietary caffeine levels and longer telomere length suggests that caffeine consumption during pregnancy may have positive long-term implications from a chromosomal perspective.

- There is a need for more studies to further explore the effect of caffeine consumption during pregnancy on fetal telomere length.

\section{References}

I. Salihu HM, King LM, Nwoga C, et al. Association Between Maternal-Perceived Psychological Stress and Fetal Telomere Length. Southern Medical Journal. 2016; 109. I2: 767-72.

2. Saiardi A, Resnick AC, Snowman AM, Wendland B, and Snyder SH. Inositol Pyrophosphates Regulate Cell Death and Telomere Length through Phosphoinositide 3-kinase-related Protein Kinases. Proceedings of the National Academy of Sciences. 2005; 102.6: $|9| 1-9 \mid 4$.

3. Liu JJ, Crous-Bou M, Giovannucci E, and De Vivo I. Coffee Consumption is Positively Associated with Longer Leukocyte Telomere Length in the Nurses' Health Study. Journal of Nutrition. 2016; 146(7): 1373-378.

4. Tucker LA. Caffeine Consumption and Telomere Length in Men and Women of the National Health and Nutrition Examination Survey (NHANES). Nutrition \& Metabolism. 2017; 14 (I).

5. Jarosz M, Wierzejska R, Siuba M. Maternal Caffeine Intake and its Effect on Pregnancy Outcomes. European Journal of Obstetrics \& Gynecology and Reproductive Biology. 2012; 160.2:156-160

6. Wierzejska, R, Jarosz M, Siuba M, and Sawicki WA. Comparison of Maternal and Fetal Blood Levels of Caffeine and its Metabolite. A Pilot Study. Polish Gynaecology. 2014;85.7.

7. Vik T, Bakketeig L, Trygg KU, Lund-Larsen K, and Jacobsen G. High Caffeine Consumption in the Third Trimester of Pregnancy: Gender-specific Effects on Fetal Growth. Paediatric and Perinatal Epidemiology. 2003; 17.4: 324-31.

8. World Health Organization (WHO). Restricting Caffeine Intake during Pregnancy. World Health Organization. https://www.who.int/elena/titles/ caffeine-pregnancy/en/Accessed II Mar. 2017.

9. Rhee J, Kim R, Kim Y, et al. Maternal Caffeine Consumption during Pregnancy and Risk of Low Birth Weight: A Dose-Response Meta-Analysis of Observational Studies. Plos One. 20I5; I0.7.

10. Adams T, Kelsberg G, and Safranek S. Clinical Inquiry: Does Caffeine Intake during Pregnancy Affect Birth Weight? The Journal of Family Practice. Mar. 2016.

II. Li J, Hong Zhao J, Zhang J, Tang Y, and Xin C.A Metaanalysis of Risk of Pregnancy Loss and Caffeine and 
Coffee Consumption during Pregnancy. International Journal of Gynecology \& Obstetrics. 20 I 5; | 30.2: I | 6-22.

12. National Institutes of Health. Diet History Questionnaire, Version I.0. National Institutes of Health,Applied Research Program. 2007.

13. National Cancer Institute. Diet*Calc Analysis Program, Version 1.4.3. National Cancer Institute, Applied Research Program. 2005.

14. National Cancer Institute. DHQ Nutrient Database. dhql_003282006.csv. National Cancer Institute, Applied Research Program. 2005.

15. Subar AF, Ziegler RG, Thompson FE, Johnson CC, Weissfeld JL, Reding D, et al. For the Prostate, Lung, Colorectal, and Ovarian Cancer Screen Trial Investigators. is shorter always better? Relative importance of questionnaire length and cognitive ease on response rates and data quality for two dietary questionnaires. American Journal of Epidemiology. 200 I; I53(4):404.

16. Thompson FE, Subar AF, Brown CC, Smith AF, Sharbaugh CO, Jobe JB, et al. Cognitive research enhances accuracy of food frequency questionnaire reports: results of an experimental validation study.
Journal of the American Dietetic Association. 2002; 102:212-25.

17. Subar AF, Thompson FE, Kipnis V, Midthune D, Hurwitz P, McNutt S, et al. Comparative Validation of the Block,Willett, and National Cancer Institute Food Frequency Questionnaires: The Eating at America's Table Study. American Journal of Epidemiology. 200I; I54:1089-99.

18. Drury S, Esteves K, Hatch V. et al. Setting the Trajectory: Racial Disparities in Newborn Telomere Length. The Journal of Pediatrics. 20I5; 166 (5): II8I-II86.

19. Okuda K, Bardeguez A, Gardener JP. et al. Telomere Length in the Newborn. Pediatric Research. 2002; 52 (3): 377-38I.

20. Prescott J, M. Du. Wong J, and De Vivo I. Paternal Age at Birth is Associated with Offspring Leukocyte Telomere Length in the Nurses' Health Study. Human Reproduction. 2012; 27.12: 3622-63I.

21. The World's Top Drink. National Geographic. https:// www.nationalgeographic.com/people-and-culture/ onward/20 I4/04/28/the-worlds-top-drink/Accessed June 72019. 